Article DOI: https://doi.org/10.35219/ann-ugal-math-phys-mec.2019.1.15

\title{
PROPERTIES AND APPLICATIONS OF CONDUCTING POLYMER NANOCOMPOSITES AS ELECTRODE MATERIALS FOR SUPERCAPACITORS
}

\author{
Radu Costin Pană ${ }^{1}$ \\ ${ }^{1}$ University of Bucharest, Faculty of Physics, Bucharest, Romania, email: radu.pana@gmail.com
}

\begin{abstract}
Supercapacitors applications can be seen in many proficient used electronics, especially in highutilization equipment and industrial specific tasks. They are capable to fill power-supply gaps between regular capacitors with considerable power output and batteries owning high energy per mass. Hence the requirements for long endurance supercapacitors developed for complex standalone energy storages, microelectronics and sensors among other devices. The polymer nanocomposite polyaniline (PANI) and the spin-off polypyrrole by-product polyethylenedioxythiophene (PEDOT) as electrode materials for supercapacitors make the objects of this study. The investigation of the aforementioned modified nanostructures, in order to obtain valuable informations, was done by scanning electron microscopy and Raman study. Supercapacitors qualities make them the best solution when it comes to applications that require great number of quick charge-discharge cycles, as regenerative braking, burst-mode operations and energy storage on limited periods of time. Nanostructure composites as electrode materials represent the future in terms of safeness and costs for chemical ultracapacitors. This review underlines the prospective qualities and properties regarding polymer composites electrode material.
\end{abstract}

Keywords: polymer nanocomposites, electrode materials, supercapacitors.

\section{INTRODUCTION}

A supercapacitor is a special technique capacitor that provides power density and intermediate energy density between conventional electrolytic capacitors and batteries.

Composed of several cells connected in series-parallel, they allow a high voltage and a high output current (power density of the order of several $\mathrm{kW} / \mathrm{kg}$ ) and store a quantity of intermediate energy between the two storage modes mentioned above and can restore it faster than a battery. They are therefore often used as a backup energy storage element, in addition to batteries or a fuel cell. In particular, they have the advantage of being effective at very low temperatures.

The supercapacitor is composed of two porous electrodes, generally made of activated charcoal and impregnated with electrolyte, which are separated by an insulating and porous membrane (to ensure ionic conduction). The electric double layer develops on each electrode-electrolyte interface, so that a supercapacitor can be seen schematically as the combination of two capacitors in series, one to the positive electrode and the other to the electrode negative. The mobility of the anions, much less hydrated, is greater than that of the cations. They move more easily in the structure of the activated carbon and form a layer of smaller thickness, so that an anode capacitance value higher than that of cathode is observed. Due to the capacitor association laws, the capacitance of the series set is 
always less than the lower of these two capacities. It is also for this reason that the supercapacitor is polarized, each electrode being optimized for either anions or cations.

Supercapacitors applications can be seen in many proficient used electronics, especially in high-utilization equipment and industrial specific tasks. They are capable to fill power-supply gaps between regular capacitors with considerable power output and batteries owning high energy per mass. Hence the requirements for long endurance supercapacitors developed for complex stand-alone energy storages, microelectronics and sensors among other devices. The polymer nanocomposite polyaniline (PANI) and the spin-off polypyrrole by-product polyethylenedioxythiophene (PEDOT) as electrode materials for supercapacitors make the objects of this study.

\section{FABRICATION METHOD}

To prepare mixed metal oxide nanocomposites, various synthesis methods including solid state reactions (i.e. thermal decomposition of mechanical mixtures of metal salts), mechanical mixing of metal oxides (i.e. ball milling), and chemical co-precipitation and electrochemical anodic deposition from solutions containing metal salts, have been used.

As a conducting polymer that has been used to composite with metal oxides to form electroactive materials we utilized Poly 3,4-ethylenedioxythiophene (PEDOT). PEDOT is a stable and environmentally friendly polymer and has controllable electrical conductivity [1]. However, PEDOT suffers from problems such as volumetric swelling and shrinkage during the insertion and ejection of ions [2]. PEDOT was comprised with pseudocapacitive metal oxides such as $\mathrm{MnFe}_{2} \mathrm{O}_{4}, \mathrm{CoFe}_{2} \mathrm{O}_{4}$ and $\mathrm{MoO}_{3}$ to improve its property. The synergistic effect of composite formation plays a significant role to increase the capacitance value [3].

Poly 3,4-ethylenedioxythiophene (PEDOT) nanocomposites was created by adding 3,4ethylenedioxythiophene monomer into a lithium molybdenum nanoparticle suspension, and afterwards, Iron chloride $\left(\mathrm{FeCl}_{3}\right)$ was added to the composite as the oxidizing agent under microwave hydrothermal conditions in order for the polymerization to occur.

\section{INVESTIGATION METHODS}

Scanning electron microscopy (SEM) and Raman study on PEDOT was conducted to derive information regarding the Raman shift, and absorption bands of inter and/or intra-gap states.

The Raman spectrum of the PEDOT nanocomposite was obtained by depositing the film on a silicon substrate. Specific surface areas were used as analytical techniques to characterize the morphologies, structures, chemical composition and surface area of nanocomposite electroactive materials as shown in Fig. 1.

Fig.2(a) shows the SEM picture of PEDOT nanofibers. It shows the 100-200 nm thick PEDOT fibers. The SEM image in Fig. 2(b) depicts the wrinkled morphology of PEDOT film. The nanofibers were afterwards doped using $1 \mathrm{M} \mathrm{HCl}$ for a couple of minutes. Fig. 2(c) shows that the $\mathrm{HCl}$ doped, and as well the fact that the PEDOT film has a higher surface roughness as well as a wide pore size distribution areas.

The conductivity performance of PEDOT nanocomposites for electrochemical capacitor has been investigated using an electrochemical kit. The electrochemical measurements were recorded at room temperature in house cell testing unit comprising of PEDOT as working electrode and counter electrode, in $2 \mathrm{M} \mathrm{H}_{2} \mathrm{SO}_{4}$ and $2 \mathrm{M} \mathrm{HCl}$. The cathodic peak $(0.25 \mathrm{~V})$ has changed from negative to positive value and the anodic peak at $0.59 \mathrm{~V}$ has shifted toward left, increasing the potential sweep rates from 10 to $100 \mathrm{mV} / \mathrm{s}$. 
Fig. 1. Raman spectrum of PEDOT nanocomposite

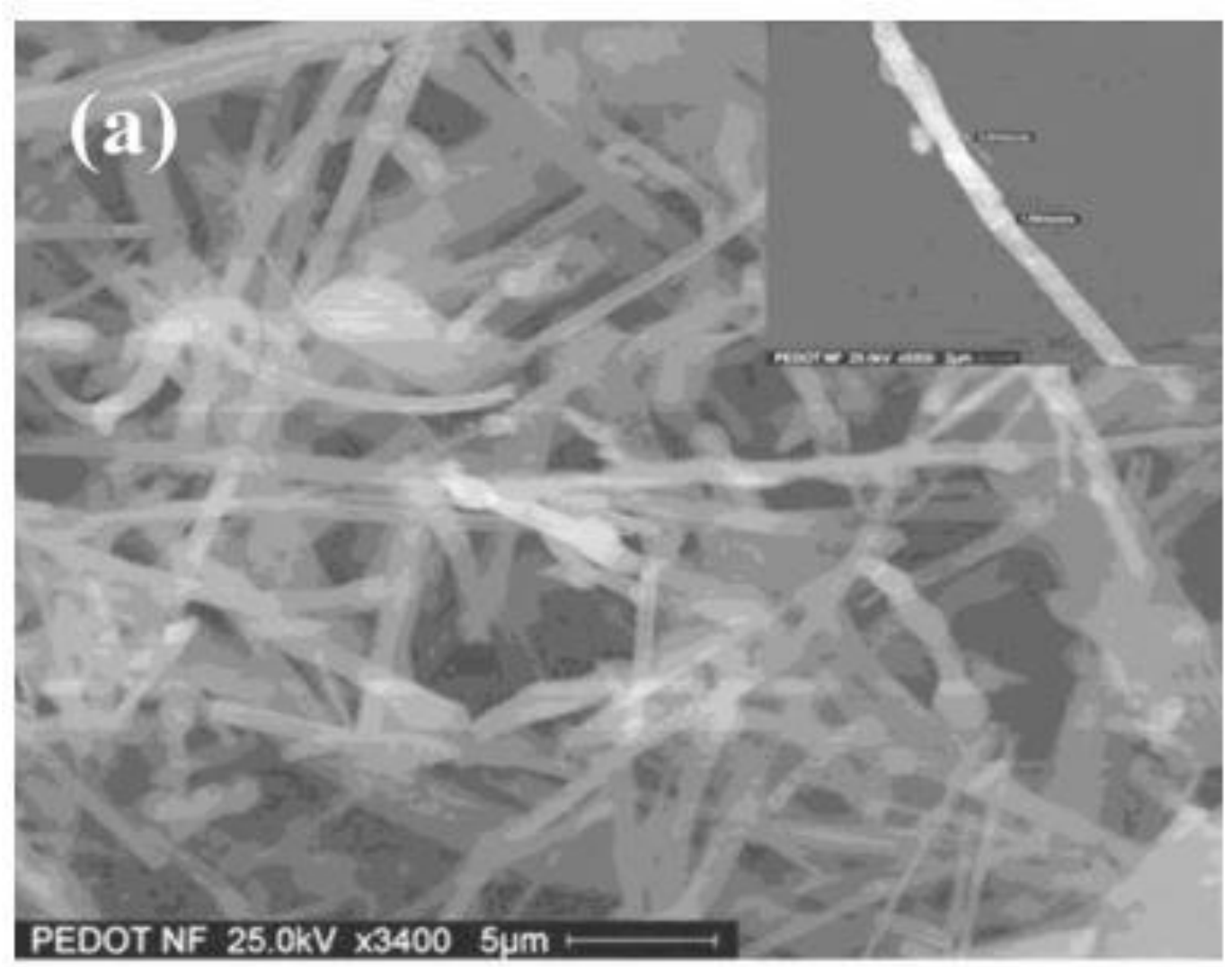




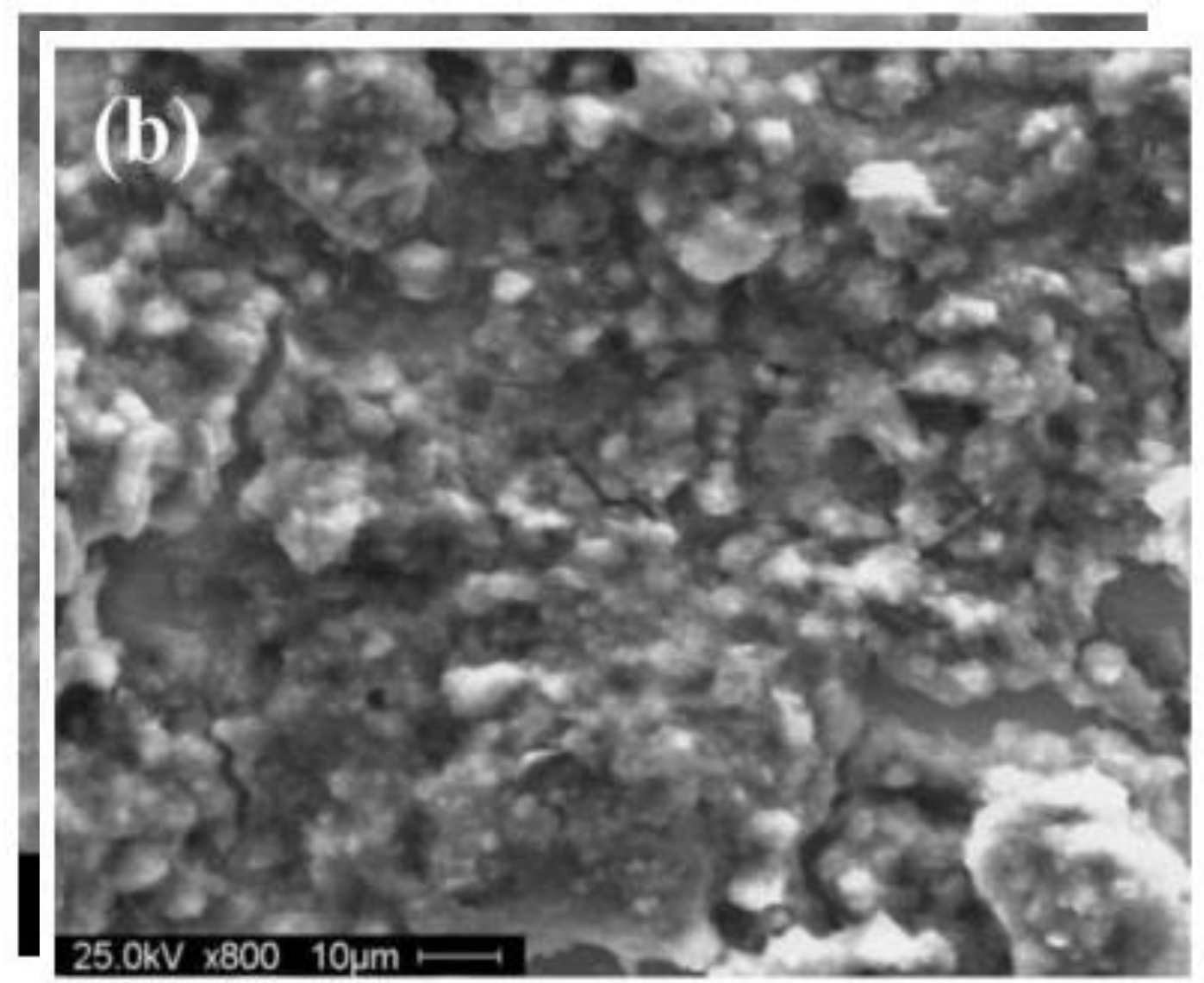

Fig. 2. SEM pictures of: (a) PEDOT, (b) un-doped PEDOT, and (c) doped PEDO

\section{CONCLUSIONS}

Nanocomposite electroactive materials that have been developed so far have demonstrated huge potential for supercapacitor applications. It has been shown that significant improvement in term of specific surface area, electrical and ionic conductivities; specific capacitance, cyclic stability, and energy and power density, of supercapacitors can be achieved by using nanocomposite electroactive materials. This can be attributed to the complementary and synergy behaviours of the consisting material components, the unique interface characteristics and the significant increase in surface areas, as well as nano-scale dimensional effects.

\section{References}

1. Wang J., Xu Y., Chen X., Du X., Electrochemical supercapacitor electrode material based on poly(3,4-ethylenedioxythiophene)/polypyrrole composite, J. Power Sources, 163(2), pp. 11201125, 2007.

2. Kim H., Popov N., Synthesis and Characterization of MnO2Based Mixed Oxides as Supercapacitors, Journal of the Electrochemical Society, 150(3), pp. 56-62, 2003.

3. Ryu K. S., Lee Y. G., Hong Y. S., Park Y. J., Wu X., Kim K. M., Kang M. G., Park N. G., Chang S. H., Poly(ethylenedioxythiophene) (PEDOT) as polymer electrode in redox supercapacitor, Electrochimica Acta, 50(2-3), pp. 843-847, 2004. 